\title{
Children Use Artifacts to Infer Others' Shared Interests
}

\author{
Madison L. Pesowski (mpesowski@ucsd.edu) \\ University of California, San Diego, Department of Psychology \\ 9500 Gilman Drive, San Diego, CA 92093-0109 USA \\ Deborah Kelemen (dkelemen@bu.edu) \\ Boston University, Department of Psychological \& Brain Sciences \\ 64 Cummington Mall, Boston, MA 02215 USA \\ Adena Schachner (schachner@ucsd.edu) \\ University of California, San Diego, Department of Psychology \\ 9500 Gilman Drive, San Diego, CA 92093-0109 USA
}

\begin{abstract}
Artifacts - the objects we own, make, and choose - provide a source of rich social information. Adults use people's artifacts to judge others' traits, interests, and social affiliations. Here we show that 4-year-old children $(\mathrm{N}=32)$ infer others' shared interests from their artifacts. When asked who had the same interests as a target character, children chose the character with a conceptually similar object to the target's - an object used for the same activity - over a character with a perceptually similar object. When asked which person had the same arbitrary property (bedtime, birthday, or middle name), children did not systematically select either character, and most often reported that they did not know. Adults $(\mathrm{N}=32)$ made similar inferences, but differed in their tendency to use artifacts to infer friendships. Overall, by age 4, children show a sophisticated ability to make selective, warranted inferences about others' interests based solely on their artifacts.
\end{abstract}

Keywords: social cognition; cognitive development; social inferences; shared preferences; artifacts; ownership

\section{Introduction}

Children grow up surrounded by human-made objects, including tools and toys (Kelemen \& Carey, 2007; Pugh, 2009). These artifacts are useful not only as tools, but also as a source of social information: Adults interpret the artifacts of others as cues to their identities, and intentionally use artifacts as social signals, actively choosing and displaying certain artifacts to shape others' perceptions of them (Belk, 1988; Kleine et al., 1995; Solomon, 1983; Wheeler \& Bechler, 2021). Based solely on the artifacts a person owns, adults form quick and accurate judgments about a person's traits, interests, and social affiliations (e.g., Gosling, 2008; Richins, 1994). Overall, artifacts appear to play an important role in adults' social reasoning.

How does artifact-based social reasoning develop in childhood? In the current work, we explore children's ability to infer shared or common interests between other people from the artifacts they choose and own. This ability is important because children are often tasked with navigating novel social environments. Inferring common interests from others' artifacts could help children successfully initiate conversations with new social partners, and allow them to understand relationships between peers.

\section{The Development of Artifact-Based Social Reasoning}

Several cognitive foundations for artifact-based social reasoning are in place in early childhood, including an understanding of ownership (e.g., Nancekivell, Van de Vondervoort \& Friedman, 2013) and the relationship of choice to preference (Diesendruck et al., 2015; Jara-Ettinger et al, 2015; Pesowski, Denison, \& Friedman, 2016). Young children can also infer which individuals share preferences from non-artifact-based information, like who previously interacted prosocially (Afshordi, 2019).

Can children use objects to infer relationships between other people, by considering how objects reflect ways in which others may be similar? Young children can use objects to relate other people to themselves: For instance, after observing two people choose objects from pairs of toys, 2year-olds copied the choices of the person who had previously chosen interesting toys when selecting between visually inaccessible toys and books for themselves - thus generalizing the idea that they tended to share preferences with that person (Fawcett \& Markson, 2010a). By age 3, children also choose to play with someone who shares their toy preferences (e.g., likes dinosaurs), and not someone who has an arbitrary similarity (e.g., a similar-looking sticker; Fawcett \& Markson, 2010b).

However, for artifact-based social reasoning to inform understanding of relationships between peers, children would need to use objects to reason about relations between multiple other people. The computations required for this process may be more complex than for detecting maximum similarity to the self: Knowing one's own mental states (such as preferences) is cognitively easier, and develops earlier, than reasoning about the mental states of others (e.g., Wellman, 2014). In addition, children initially use some social attributes like gender only in first-person reasoning, and only later use them for third-person inferences (Shutts, Pemberton Roben, \& Spelke, 2013). Thus, the development of children's third-party inferences from artifacts may not parallel their reasoning about who shares their own interests and preferences. 
Here we ask whether young children infer whether other people have shared interests based on the artifacts (toys) that they choose and own. In doing so, we aim to characterize the type of cognitive processes children use to draw conclusions about shared interests: A simple strategy based on matching perceptual features of artifacts, or a more complex process of explanation-based reasoning.

\section{Two Accounts of Children's Reasoning}

We hypothesize that children will draw conclusions about shared interests through explanation-based reasoning, as an inference to the best explanation (Lipton, 2004; Tenenbaum, Griffiths, \& Kemp, 2006). Under this account, to explain observed data (e.g., two people have similar artifacts), we consider multiple hypotheses, and then choose the one that offers the best explanation (e.g., they both enjoy the activity the objects are used for). This type of inferential reasoning occurs in childhood in multiple related domains, including causal induction, and reasoning about others' mental states (Baker, Saxe, \& Tenenbaum, 2009; Jara-Ettinger et al., 2015; Pesowski et al., 2020).

This account makes the prediction that children should infer that two people have shared interests when they own or choose similar artifacts, and crucially should privilege conceptual similarity over perceptual features. That is, if two people have objects that are used for the same kind of activity, but look different (e.g., a baseball and a catcher's mitt), one might infer that they both share an interest in that activity (e.g., baseball). However, if two people have objects that look similar, but are not used for the same kind of activity (e.g., a baseball and a beachball), this would not imply a shared interest, despite the perceptual resemblance.

There are two reasons to suspect that children may prioritize artifacts' conceptual similarity in this way when making social inferences. First, children readily reason about objects' functions: They categorize and label objects by their intended function rather than by their perceptual features (e.g., Gelman \& Bloom, 2000; Kelemen, 1999; Kemler Nelson et al., 2000; Träuble \& Pauen, 2007), and use artifacts' properties to reason about their intended purpose (Kelemen, Seston, \& Saint Georges, 2012). Second, children are able to prioritize conceptual similarities over perceptual similarities in some contexts, for example to infer which natural kinds (animals, rocks) are similar to each other (Gelman \& Markman, 1986).

However, it is also plausible that young children may use perceptual similarity as a heuristic, and ignore relevant conceptual information. Since the work of Piaget, it has been argued that young children use heuristics and superficial features in this way, rather than engaging in deeper reasoning (e.g., Jones \& Smith, 1993; Piaget, 1929; Sloutsky, Kloos, \& Fisher, 2007). Over the preschool years, children have been argued to shift away from prioritizing perceptual information toward prioritizing conceptual information, as in the case of the shape-to-taxonomic shift in word meaning extensions from ages 3-5 (Imai, Gentner, \& Uchida, 1994).
If young children use objects' perceptual features to make social inferences, children may note the perceptual similarity of object pairs, and infer that owners of similar-looking objects are more similar to one another in other dimensions as well. This should lead to specific errors when objects' conceptual information and perceptual similarity are pitted against one another: Children's decisions should flip, such that they pick the perceptual match over the conceptual match in this case.

\section{The Current Study}

To test these two accounts of children's reasoning, we introduced children to three characters (a target and two others), and a toy each one had brought from home to play with after school. We manipulated whether the target's toy was conceptually similar to the other characters' toys (in the sense of being used for the same kind of activity), and/or perceptually similar to the other characters' toys. In half of the trials, one toy was both conceptually and perceptually more similar to the target's toy than the other - i.e., the conceptual and perceptual cues were congruent. In the other half of trials, these cues were incongruent: One toy was conceptually similar to the target's, while the other toy was perceptually more similar (i.e., it was of similar color and shape). If children use relevant conceptual information to reason about shared interests, then children should answer that the character with a conceptually similar toy shares the target's preferences, even on trials where this toy is more perceptually distinct.

We also asked whether children make specific, warranted inferences about shared interests, or over-generalize by inferring that people with similar artifacts are more similar on any dimension. To test this, we asked children two kinds of questions: Questions about relevant topics (liking the same games, same activities, or being best friends), and other, irrelevant dimensions (having the same bedtime, birthday, or middle name). If children make specific reasoned inferences about activity preferences, they should choose the character with the similar toy only when answering questions about relevant topics, not similarly-worded but irrelevant questions. However, if children always use artifacts' similarity in their judgements, then they should make consistent judgments regardless of the type of social inference they are asked to make. We included the option for children to answer "I don't know" on all trials, which pilot testing suggested improved children's engagement with the task by minimizing frustration (see Method).

To better understand the reasoning behind children's inferences, we also asked children to explain their judgments. Because children younger than age 4 often have difficulty providing adequate justifications (e.g., Kenward \& Dahl, 2011; Terrier et al., 2016; Van de Vondervoort \& Hamlin, 2017), we tested 4-year-old children in the current study. We also chose 4-year-olds because at this age, children have a sophisticated understanding of the artifact domain, particularly about artifacts' intended functions (e.g., Kelemen $\&$ Carey, 2007), and their reasoning in other domains appears 
to be actively shifting from using perceptual features to conceptual information (e.g., a shape-to-taxonomic shift in word meaning extensions, from age 3-5; Imai et al., 1994).

We also tested adults as a point of comparison, to determine whether children's inferences resemble those of adults. If children and adults use explanation-based reasoning to infer shared interests from artifacts, then both populations should make only relevant and warranted social inferences, and privilege conceptual over perceptual similarity. In contrast, if 4-year-old children have not yet shifted from prioritizing perceptual information in this domain, then there should be notable differences between children's and adults' social inferences, with children always selecting the character with the perceptually similar toy.

The experimenter script, sample images, data, and analysis code are available at https://osf.io/tnv5z

\section{Methods}

\section{Participants}

32 four-year-old children $\left(M_{\mathrm{age}}=4\right.$ years; 6 months, range $=$ $4 ; 1-4 ; 10,16$ females $)$ and 32 adults $\left(M_{\mathrm{age}}=20.5\right.$ years, range $=$ 18-29 years, 22 females) participated. Children were recruited from the metro-Boston area from birth lists and local community events. One additional child participant was excluded from the final sample due to lack of attention and failure to engage in the task. Adults were recruited from the undergraduate student population at a large public university in California, and earned course credit in exchange for participation. One additional adult participant was tested but excluded for failing an attention check question, which asked participants to identify which one of several questions they had not been asked during the task.

In addition, to validate our stimuli, we recruited a separate sample of adults online using Prolific $\left(\mathrm{N}=40 ; M_{\mathrm{age}}=31\right.$ years, range $=18-59$ years, 20 female, 1 additional gender identity). These participants were asked to judge which toy was more perceptually similar to the target in each set (see Stimuli).

\section{Design}

The experiment used a $2 \times 2$ within-subject design (trial type: conceptual and perceptual similarity congruent vs. incongruent; question type: relevant vs. irrelevant). Children completed 6 trials: 3 congruent and 3 incongruent. The location of the non-target objects (left, right) and which type of question was asked first (relevant, irrelevant) was counterbalanced. There were three possible relevant questions (likes same activities, games, best friends); and three irrelevant questions (same bedtime, birthday, middle name). Each of the six questions occurred twice, once in each type of trial (congruent, incongruent), and were presented in one of four different pseudo-random orders. The 3 congruent trials were always presented first, followed by the 3 incongruent trials. Within each trial, all characters were of the same gender; half of the trials had male characters and the other half had female characters.

\section{Stimuli}

Stimuli consisted of cartoon images of children, each paired with a photograph of a toy or set of toys. The image of the target character and toy was placed above the two other characters, centered and equidistant. On congruent trials, the target's toy was conceptually and perceptually more similar to one of the character's toys than other (see examples in Figure 1). On incongruent trials, one of the toys was conceptually similar to the target's, while the other toy was conceptually different but perceptually more similar (i.e., was the same shape and color as the target). The three incongruent stimulus sets were: A set of paints (target), paintbrushes, xylophone (similar colors and orientation to paints); Teapot (target), teacups, watering can (similar shape and color to teapot); Toy stethoscope (target), toy band aids, headphones (similar shape and color to stethoscope).

To validate the idea that the perceptually similar toy looked most similar to the target's toy, an independent group of adults were shown the same triads of toys, and asked: "Which of the two objects below looks most similar (visually similar) to the object above?". Participants near-unanimously agreed with our designations, selecting the perceptually similar toy as looking most similar to the target $(M=97.92 \%$, $\mathrm{SEM}=.44 \% ; 235 / 240$ total trials across 40 participants). This was the case both in the congruent trials $(M=100 \%, \mathrm{SEM}=0$; $120 / 120$ total trials) and also in the incongruent trials $(M=95.83 \%, \mathrm{SEM}=1.23 \% ; 115 / 120$ total trials $)$, validating the idea that these items actually were perceptually more similar, as intended by our manipulation.

\section{Procedure}

Children were tested individually in the laboratory, seated at a small table next to the experimenter. Children first took part in a brief training session regarding the "I don't know" option, in which they were introduced to an icon of a character shrugging (gender-matched to the participant; see Figure 1) and encouraged to use this icon in response to a question where the answer was unknown (what was inside a closed box).

On each trial, the experimenter pointed to the images, and said: "This is $[\mathrm{X}]$, and [s/he] goes to kindergarten at Smith Elementary School. [X]'s teacher told all the children in the class to bring something in from home, to play with after school. This is what $[\mathrm{X}]$ brought in to play with after school. Here are two other children who are in the same class as [X], and here are the things they brought in to play with after school". To ensure that children were familiar with the objects, they were asked to identify and label the toys. If they did not know, they were asked if they knew what the toy was for; if not, the experimenter labeled the object and stated its function. This occurred only rarely (12 out of 576 object presentations).

On each trial, children were asked one relevant and one irrelevant question. All questions took the same form: "Who do you think [likes/does/has] the same [thing] as [Target]: [character A], [character B], or you don't know?". For example, "Who do you think likes to play the same kinds of 


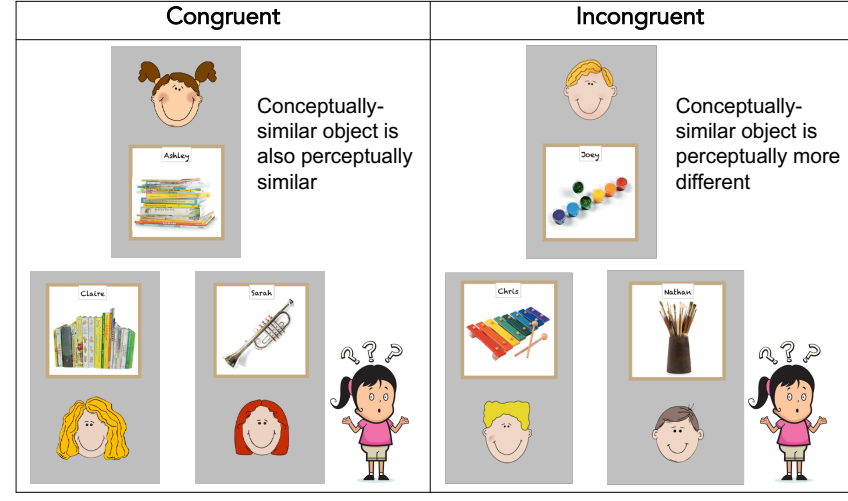

Figure 1: Methods. Sample images used in the test trials. The top character was the target; children answered by selecting one of three options: the character on the left, the other character, or "I don't know" (represented by the character on the right). In congruent trials (left panel), the target object was conceptually and perceptually similar to one of the objects; whereas in the incongruent trials (right panel), the target object was conceptually similar to one object, but perceptually similar to the other.

games as [Target]...?"; "Who do you think has the same birthday as [Target]...?". Children responded by indicating one of the characters or by saying "I don't know", either verbally or by pointing. Trials on which children responded "both" or "neither" were excluded from analysis; this occurred only rarely (6/192 trials). If children responded by indicating one of the characters, they were asked to explain their answer ("Why do you think that?").

Adults were tested using a similar task, adapted for adult participants and for online testing. Adults did not receive training on how to respond "I don't know", were not asked to label objects, and were not asked to explain their answers. Adults saw the same images, with vignettes and questions presented as text, and the "I don't know" response option also presented as text.

\section{Coding of Children's Explanations}

Two coders naïve to the hypotheses and children's judgments independently coded children's explanations of their answers into four non-mutually-exclusive categories: toy-based, character-based, I don't know, and Other. Toy-based explanations referred to the toys' similarity, the toys themselves, or stated that the characters like or would engage in the activity the toys were for (e.g., "they both have soccer balls", "because she has books"). Character-based explanations appealed to the characters' features (e.g., faces, hair, names). The 'Other' category encompassed justifications that were irrelevant, ambiguous, or restated the question. Two explanations were coded into more than one category, and children failed to provide an explanation on 5 trials. Intercoder reliability was $98.24 \%$, and disagreements were resolved by a third coder, who remained unaware of the other coders' judgements.

\section{Results}

To test whether children made specific, warranted inferences about others' shared interests from their artifacts, we used a logistic regression to predict children's selection of the character with the conceptually similar toy, with the predictors of trial type (congruent/incongruent), question type (relevant/irrelevant), the interaction of these two factors, and subject (as a random factor). As predicted, there was a main effect of question type (nested model comparison, $\left.\chi^{2}(1)=38.06, p<.001\right)$, such that children were more likely to choose the agent with the conceptually similar toy when asked relevant questions $(M=59 \%, \mathrm{SEM}=4 \%)$ than irrelevant questions $(M=29 \%, \mathrm{SEM}=3 \%)$. Follow-up analyses showed that when answering relevant questions, children chose the character with the conceptually similar toy at a rate greater than chance (out of 3 options, chance $=.33 ; t(188)=7.17$, $p<.001)$; and selected this character at chance when answering irrelevant questions $(t(188)=1.34, p=18)$. There was no main effect of trial type, such that children chose the character with the conceptually similar toy at similar rates in congruent vs. incongruent trials $\left(\chi^{2}(1)=3.25, p=.07\right)$. However, there was a significant interaction between trial type and question type $\left(\chi^{2}(1)=6.88, p=.009\right)$ : When answering relevant questions, children chose the character with the conceptually similar toy more often on trials with congruent cues $(M=69 \%, \mathrm{SEM}=5 \%)$ than incongruent cues $(M=48 \%$, SEM=5\%); see Figure 2a.

We used a separate logistic regression model to test when children tended to answer "I don't know", with the same predictors. As predicted, there was a main effect of question type $\left(\chi^{2}(1)=20.33, p<.001\right)$, with children being more likely to indicate they did not know in response to irrelevant questions $(M=43 \%, \mathrm{SEM}=4 \%)$ than relevant questions $(M=24 \%$, SEM=3\%). Follow-up analyses showed that children answered that they did not know at rates below chance when responding to relevant questions (3 options, chance $=.33 ; 24 \%, t(188)=2.96, p=.004)$, and at rates greater than chance when answering irrelevant questions $(43 \%$, $t(188)=2.87, p=.005)$. Neither trial type (nested model comparison, $\left.\chi^{2}(1)=.78, p=.38\right)$ nor the interaction between trial and question type $\left(\chi^{2}(1)=2.46, p=.11\right)$ predicted the tendency to answer "I don't know".

\section{Differences by Question Topic}

To determine if children's responses differed by specific question topic (activities, games, friendship; bedtime, birthday, middle name), we also performed a logistic regression within each question type (relevant/irrelevant), with the predictors of specific question topic and subject (as a random factor). Children selected the character with the conceptually similar toy at equal rates when asked who liked the same games and activities (71\% and 64\% respectively, $p=.33$, logistic regression with activities as the reference level). However, children were less likely to select this character when inferring friendship $(40 \%, \beta=-1.19, p=.004)$. When answering irrelevant questions, children selected the character with the conceptually similar toy at equal rates 
when asked who had the same bedtime or birthday $38 \%$ and $27 \%$ respectively, $p=.17$, logistic regression with bedtime as the reference level); however, they were less likely to select this character when asked who had the same middle name $(21 \%, \beta=-.92, p=.03)$; see Figure $3 a$.

\section{Comparison to Adult Reasoning}

In most regards, adults' answers patterned with children's, with more consistency across participants (see Figures 2b, 3b). A logistic regression predicting adults' selection of the character with the conceptually similar toy, with the predictors of trial type, question type, and subject (as a random factor), revealed main effects of trial type $\left(\chi^{2}(1)=13.14, p<.001\right)$, such that adults were more likely to select this character on congruent than incongruent trials (55\% vs. $44 \%$ ); a main effect of question type $\left(\chi^{2}(1)=242.12\right.$, $p<.001)$, with adults selecting this character more in response to relevant than irrelevant questions ( $84 \%$ vs. $15 \%)$; and no interaction between trial and question type $\left(\chi^{2}(1)=.30\right.$, $p=.58)$. Adults were more likely than children to select the character with the conceptually similar toy when asked relevant questions ( $83 \%$ vs. $59 \%, \beta=-1.44, p<.001$; logistic regression, data from relevant questions only; predictors of age group, and subject as a random factor). Similar to children, adults selected the character with the conceptually similar toy at equal rates when inferring who liked the same games and activities as the target (97\% and $91 \%$ respectively,
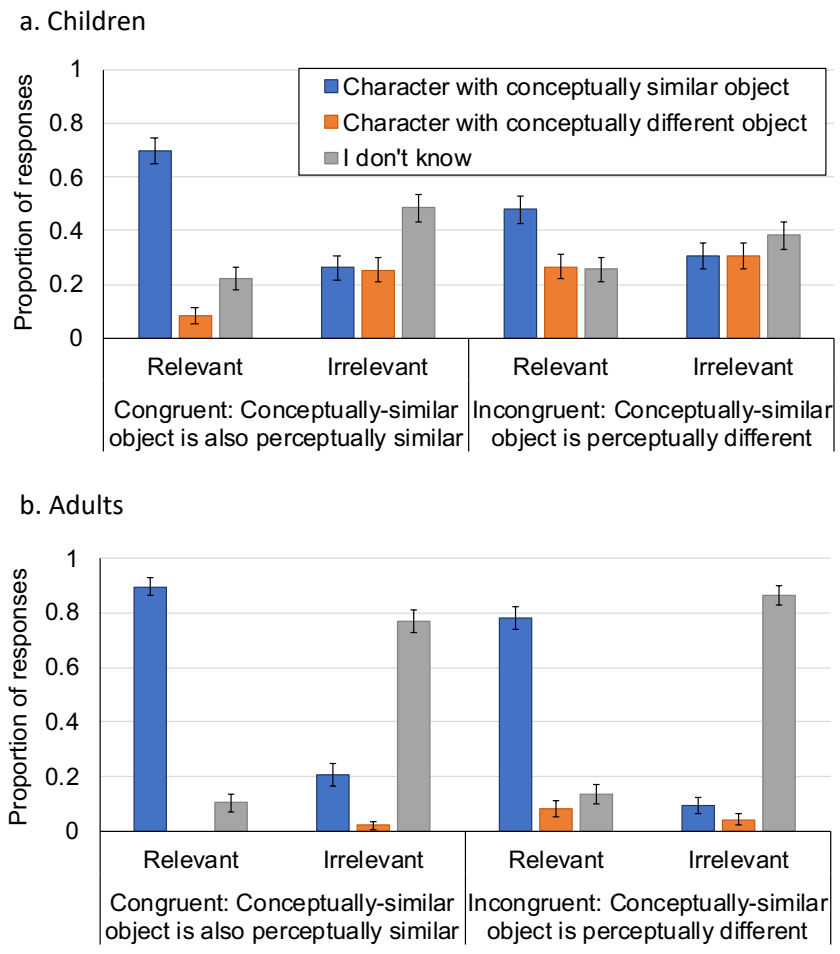

Figure 2: Results. Children's (a) and adults' (b) mean proportion scores by question type (relevant/irrelevant), trial type (congruent/incongruent), and response chosen (see legend).

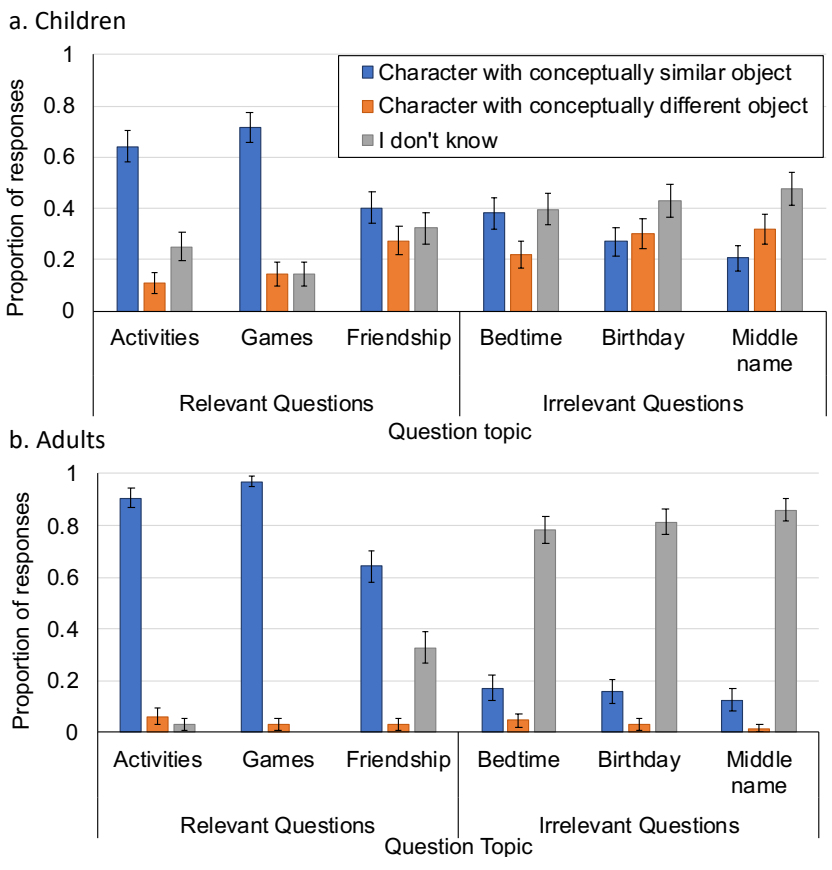

Figure 3: Results broken down by question topic. Children's (a) and adults' (b) mean responses (see legend).

$p=15$, logistic regression with activities as the reference level), and were less likely to select this character when inferring friendship $(64 \%, \beta=-1.93, p<.001)$. Notably, adults were more likely than children to infer that the character with the conceptually similar object was friends with the target ( $64 \%$ vs. $40 \%, \beta=-1.03, p=.01$ ); see Figure $3 b$.

\section{Children's Explanations}

The most common type of explanation children provided for their answers were toy-based justifications (108 out of 254 justifications), followed by "I don't know" (70/254), character-based justifications (38/254), and 'other' explanations (38/254). Children were more likely to provide toy-based explanations when answering relevant questions than irrelevant questions ( 84 vs. 24 justifications, $\beta=2.31$, $p<.001$; logistic regression with predictors of trial type, question type, and subject as a random factor), and were more likely to provide character-based explanations when answering irrelevant questions than relevant ones ( 25 vs. 13 justifications, $\beta=-1.28, p=.003)$. Children were also more likely to say that they did not know why they selected a character when answering irrelevant questions than relevant questions (42 vs. 28 justifications, respectively, $\beta=-1.92$, $p<.001)$.

\section{Discussion}

We find that 4-year-old children use the artifacts people own and choose to make selective, warranted social inferences. When asked to infer who had the same interests as a target character, children chose the character with a conceptually similar object to the targets' - an object used for the same 
activity - over a character with a different object. However, children did not infer that people with similar objects were similar on every dimension: When asked which person had the same arbitrary property (bedtime, birthday, or middle name), children did not systematically select either character, and most often reported that they did not know. These findings show that preschool children draw specific, warranted conclusions about others' shared interests based on their artifacts.

Children chose the characters with conceptually similar objects as having shared interests even when another characters' object was more perceptually similar. Thus, children were able to use relevant information over irrelevant surface features, contrary to theoretical accounts of children's reasoning as superficial in nature (Piaget, 1929), and in line with evidence of deeper conceptual reasoning in childhood (e.g., Gelman \& Markman, 1986). These findings contribute to the current literature by showing that at four years of age, children's cognitive ability to prioritize conceptual over perceptual information extends beyond kind-based categorization judgments to the domain of artifact-based social reasoning.

However, there was some effect of perceptual similarity: Children's performance was more consistent when perceptual and conceptual cues were congruent than when they conflicted. To a lesser extent, this was also true in adults. This may be due to distraction: People often shift to using surface features and heuristics when judgments are made quickly, under cognitive load, or without focused attention (e.g., Gigerenzer \& Gaissmeyer, 2011).

Interestingly, children differed from adults in that they did not infer that two people with conceptually similar artifacts were best friends, despite inferring that they had shared interests. This contrasts with previous research showing that children believe that shared preferences are one of the most important characteristics of friendships (Bigelow \& Gaipa, 1975; Furman \& Bierman, 1983; also see Afshordi \& Liberman, 2021). This could be due to the question itself: We asked children to identify the "best" friend, a type of friendship for which additional evidence may be required (e.g., sharing secrets, Afshordi \& Liberman, 2021). Alternatively, 4-year-olds may need a greater quantity of evidence to infer friendship, i.e., observing that two people have many similar toys. With age, children need fewer instances of people's choices or behaviors to make social inferences (Boseovski \& Lee, 2006; Kalish, 2002; Pesowski et al., 2016). Further, children in the current study might not have believed that people's shared preference for a single activity would necessarily cause them to consistently play together, an important feature of friendships for children (Furman \& Bierman, 1983).

One potential direction for future work could be to explore artifact-based social reasoning in children with Autism Spectrum Disorder (ASD). Current interventions for ASD leave a number of residual deficits, including fewer reciprocal friendships and lower peer acceptance (e.g., Chamberlain, Kasari, \& Rotheram-Fuller, 2007). These problems are thought to stem in part from difficulties identifying shared interests and common experiences, leading to issues identifying appropriate social partners and initializing conversations on topics of mutual interest (Bauminger, 2002; Laugeson et al. 2009). Understanding whether children with ASD fail to spontaneously infer others' interests from artifacts could potentially lead to the creation of novel interventions and social skills training programs that help children with ASD use artifacts to determine others' interests via explicit, conscious reasoning strategies.

Overall, these findings show that preschool children have a sophisticated ability to draw specific, warranted conclusions about others' mutual interests based solely on their artifacts. These findings suggest that children arrive at school privy to a rich and ubiquitous source of social information, such as the clothes, bags, and toys others own. Based on these artifacts, children are able to infer others' interests and relate other people to one another. From early in development, social information from artifacts may serve as implicit social glue, providing a foundation for smooth social interaction in early life.

\section{Acknowledgments}

We thank Jamie Rhiannon Fehribach, Franco Mercado, and Allena McComas for their assistance with stimulus design and data collection. This material is based upon work supported by a National Institutes of Health (NIH) Ruth L. Kirschstein National Research Service Award from the National Institute of Child Health and Human Development to A.S. (Grant 5F32HD075570).

\section{References}

Afshordi, N. (2019). Children's inferences about friendship and shared preferences based on reported information. Child Development, 90(3), 719-727.

Afshordi, N., \& Liberman Z. (2021) Keeping friends in mind: Development of friendship concepts in early childhood. Social Development, 30, 331-342.

Baker, C.L., Saxe, R., \& Tenenbaum, J.B. (2009). Action understanding as inverse planning. Cognition, 113(3), 329349.

Bauminger, N. (2002). The facilitation of social-emotional understanding and social interaction in high-functioning children with autism: Intervention outcomes. Journal of Autism and Developmental Disorders, 32(4), 283-298.

Belk, R.W. (1988). Possessions and the extended self. Journal of Consumer Research, 15(2), 139-168.

Bigelow, B.J., \& Gaipa, J.J. (1975). Children's written descriptions of friendship: A multidimensional analysis. Developmental Psychology, 11(6), 857-858.

Boseovski, J.J., \& Lee, K. (2006). Children's use of frequency information for trait categorization and behavioral prediction. Developmental Psychology, 42(3), 500-513.

Chamberlain, B., Kasari, C., \& Rotheram-Fuller, E. (2007). Involvement or isolation? The social networks of children with autism in regular classrooms. Journal of Autism and Developmental Disorders, 37(2), 230-242. 
Diesendruck, G., Salzer, S., Kushnir, T., \& Xu, F. (2015). When choices are not personal: The effect of statistical and social cues on children's inferences about the scope of preferences. Journal of Cognition and Development, 16(2), 370-380.

Fawcett, C.A, \& Markson, L. (2010a). Children reason about shared preferences. Developmental Psychology, 46(2), 299-309.

Fawcett, C.A, \& Markson, L. (2010b). Similarity predicts liking in 3-year-old children. Journal of Experimental Child Psychology, 105(4), 345-358.

Furman, W., \& Bierman, K.L. (1983). Developmental changes in young children's conceptions of friendship. Child Development, 54(3), 549-556.

Gelman, S.A. \& Bloom, P. (2000). Young children are sensitive to how an object was created when deciding what to name it. Cognition, 76(2), 91-103.

Gelman, S.A., \& Markman, E.M. (1986). Categories and induction in young children. Cognition, 23, 183-209.

Gigerenzer, G., \& Gaissmaier, W. (2011). Heuristic decision making. Annual Review of Psychology, 62, 451-482.

Gosling, S. (2008). Snoop: What your stuff says about you. New York: Basic Books.

Imai, M., Gentner, D., Uchida, N. (1994). Children's theories of word meaning: The role of shape similarity in early acquisition. Cognitive Development, 9, 45-75.

Jara-Ettinger, J., Gweon, H., Tenenbaum, J.B., \& Schulz, L.E. (2015). Children's understanding of the costs and rewards underlying rational action. Cognition, 140, 14-23.

Jones, S.S., \& Smith, L.B. (1993). The place of perception in children's concepts. Cognitive Development, 8, 113-139.

Kalish, C.W. (2002). Children's predictions of consistency in people's actions. Cognition, 84(3), 237-265.

Kelemen, D. (1999). The scope of teleological thinking in preschool children. Cognition, 70(3), 241-272.

Kelemen, D., \& Carey, S. (2007). The essence of artifacts: Developing the design stance. In S. Laurence \& E. Margolis (Eds.), Creations of the mind: Theories of artifacts and their representation. Oxford: Oxford University Press.

Kelemen, D., Seston, R., \& Saint Georges, L. (2012). The designing mind: Children's reasoning about intended function and artifact structure. Journal of Cognition and Development, 13(4), 439-453.

Kemler Nelson, D.G., Russell, R., Duke, N., \& Jones, K. (2000). Two-year-olds will name artifacts by their functions. Child Development, 71(5), 1271-1288.

Kenward, B., \& Dahl, M. (2011) Preschoolers distribute scarce resources according to the moral valence of recipients' previous actions. Developmental Psychology, 47(4), 1054-1064.

Kleine, S.S., Kleine III, R.E., \& Allen, C.T. (1995). How is a possession "me" or "not me"? Characterizing types and an antecedent of material possession attachment. Journal of Consumer Research, 22(3), 327-343.

Laugeson, E. A., Frankel, F., Mogil, C., \& Dillon, A. R. (2009). Parent-assisted social skills training to improve friendships in teens with autism spectrum disorders. Journal of Autism and Developmental Disorders, 39(4), 596-606.

Lipton, P. (2004). Inference to the best explanation ( $\left.2^{\text {nd }} E d.\right)$. New York: Routledge.

Nancekivell, S.E., Van de Vondervoort, J.W., \& Friedman, O. (2013). Young children's understanding of ownership. Child Development Perspectives, 7(4), 243-247.

Pesowski, M.L., Denison, S., \& Friedman, O. (2016). Young children infer preferences from a single action, but not if it is constrained. Cognition, 155, 168-175.

Pesowski, M.L., Quy, A., Lee, M., \& Schachner, A. (2020). Children use inverse planning to detect social transmission in design of artifacts. In S. Denison, M. Mack, Y. Xu, \& B.C. Armstrong (Eds.), Proceedings of the 42nd Annual Conference of the Cognitive Science Society (pp. 845-851).

Piaget, J. (1929). The child's conception of the world. London: Routledge \& Kegan Paul.

Pugh, A.J. (2009). Longing and belonging: Parents, children, and consumer culture. Los Angeles: University of California Press.

Richins, M.L. (1994). Valuing things: The public and private meaning of possessions. Journal of Consumer Research, 21(3), 504-521.

Shutts, K., Pemberton Roben, C.K., \& Spelke, E.S. (2013). Children's use of social categories in thinking about people and social relationships. Journal of Cognition and Development, 14(1), 35-62.

Sloutsky, V.M., Kloos, H., \& Fisher, A.V. (2007). When looks are everything: Appearance similarity versus kind information in early induction. Psychological Science, 18(2), 179-185.

Solomon, M.R. (1983). The role of products as social stimuli: A symbolic interactionism perspective. Journal of Consumer Research, 10(3), 319-329.

Tenenbaum, J.B., Griffiths, T.L., \& Kemp, C. (2006). Theory-based Bayesian models of inductive learning and reasoning. Trends in Cognitive Sciences, 10(7), 309-318.

Terrier, N., Bernard, S., Mercier, H., Clément, F. (2016). Visual access trumps gender in 3- and 4-year-old children's endorsement of testimony. Journal of Experimental Child Psychology, 146, 223-230.

Träuble, B., \& Pauen, S. (2007). The role of functional information for infant categorization. Cognition, 105(2), 362-379.

Van de Vondervoort, J.W., \& Hamlin, J.K. (2017). Preschoolers' social and moral judgments of third-party helpers and hinderers align with infants' social evaluations. Journal of Experimental Child Psychology, 164, 136-151.

Wellman, H.M. (2014). Making minds: How theory of mind develops. New York: Oxford University Press.

Wheeler, S.C., \& Bechler, C.J. (2021). Objects and selfidentity. Current Opinion in Psychology, 39, 6-11. 